\title{
Atracción e investigación de los entornos de comercio modernos
}

Grupo de Investigación Mercadeo I+2

\section{Leonardo Ortegón Cortazar}

Julio de 2020

\section{Resumen}

Los entornos de comercio modernos también denominados grandes centros comerciales son un espacio importante en el estilo de vida de los consumidores que han evolucionado a través del mejoramiento de la experiencia de visita a través de su capacidad de atracción, en este sentido, se ha realizado una investigación de carácter documental y sistemática con el fin de identificar los factores de atracción y los tópicos de investigación. Se observa que no existe un consenso respecto a la cantidad y tipología de factores que componen la atracción de los entornos de comercio moderno, sin embargo, se pueden deducir variables de atracción comunes.

Palabras clave: Entornos de comercio moderno, factores de atracción, investigación.

\begin{abstract}
Modern shopping environments also called large shopping malls are an important space in the lifestyle of consumers that have evolved through the improvement of the visiting experience through their attractiveness, in this sense, a Documentary and systematic research to identify attraction factors and research topics. If you observe that there is no consensus regarding the number and typology of factors that make up the attraction of modern commerce environments, however, you can deduce variables of common attraction.
\end{abstract}

Key words: Modern trading environments, attraction, research. 
Los entornos de comercio modernos también denominados grandes centros comerciales son un espacio importante en el estilo de vida de los consumidores (Lucia-Palacios et al, 2020; Ortegón \& Royo, 2019; Calvo-Porral \& Lévy-Mangín, 2018) debido s su oferta de entretenimiento y ocio que trascienden la capacidad de atracción de las compras (Aliagha et al., 2015; Bäckström, 2011). En consecuencia, el estudio de estos entornos de comercio moderno es de interés para la literatura de marketing al poder incentivar la intención de visita, y por tanto mejorar su gestión competitiva (Gomes y Paula, 2017; Merrilees et al., 2016;). En esta línea, el objetivo de este trabajo es documentar la atracción e investigación de los entornos de comercio moderno analizando los factores de atracción y los tópicos de investigación que tendrán que seguir las futuras investigaciones en el campo del marketing.

Varios estudios describen la importancia de examinar la atracción e investigación de los entornos de comercio moderno a través de la optimización de la experiencia de los consumidores (Calvo-Porral \& Lévy-Mangín 2018; Gomes \& Paula, 2017; Palacios, Pérez, \& Polo, 2016; Ortegon \& Royo, 2015; Frasquet, Vallet \& Gil, 2002). El-Adly \& Eid (2015) han sugerido que los entornos de comercio moderno aportan más valor para los clientes al sentirse más atraídos por una oferta y variedad más amplia de almacenes y productos, lo que ha ocasionado que dichos entornos hayan evolucionado expandiendo sus actividades y diseño arquitectónico para incluir una amplia gama de servicios y entretenimientos.

En esta línea, frente al interés de contextualizar los factores de atracción reportados por la literatura, se ha realizado una revisión sistemática de literatura mediante un arqueo bibliográfico de revistas de impacto que figuran en las bases de datos Scopus (422) clasificando y seleccionando a juicio de los autores los artículos que, por su naturaleza y contenido, son de importancia para documentar la atracción de los entornos de comercio moderno. El proceso de revisión parte de la identificación de las palabras claves asociadas a atracción o atractividad "attract*" combinada con "mall" or "shopping center" en el campo 
de negocios, administración y contabilidad; y el campo de ciencias sociales, de tal forma que se obtuvo una ecuación de búsqueda ${ }^{1}$.

Se elaboró una tabla integradora orientada a presentar trabajos empíricos disponibles siguiendo el trabajo del mismo autor (Ortegón, 2019).), incluyendo publicaciones relevantes a través de un proceso sistemático relacionando la cantidad e identificación de los factores de atracción de los entornos de comercio moderno (ver tabla 1).

Tabla 1. Factores de atracción de centros comerciales.

\begin{tabular}{lcl}
\hline \multicolumn{1}{c}{ Estudio } & $\begin{array}{c}\text { Número de } \\
\text { factores } \\
\text { considerados }\end{array}$ & \multicolumn{1}{c}{ Factores de atracción } \\
\hline $\begin{array}{l}\text { Roux, Mahlangu \& } \\
\text { Manetje, (2020). }\end{array}$ & Tres & $\begin{array}{l}\text { Señales ambientales, señales de diseño y } \\
\text { señales sociales }\end{array}$ \\
\hline Han et al, 2019 & Cuatro & $\begin{array}{l}\text { Consumo, Entorno construido, } \\
\text { Entretenimiento, Ambiente }\end{array}$ \\
\hline $\begin{array}{l}\text { Ortegon-Cortazar, } \\
\text { (2019). }\end{array}$ & Siete & $\begin{array}{l}\text { Ambiente econatural, accesibilidad, oferta, } \\
\text { perfil de clientes, diseño físico, lujo y } \\
\text { entretenimiento. }\end{array}$ \\
\hline $\begin{array}{l}\text { Elmashhara \& Soares, } \\
\text { (2019). }\end{array}$ & Dos & $\begin{array}{l}\text { Entretenimiento, interacción social con } \\
\text { vendedores }\end{array}$ \\
\hline Hu \& Jasper (2018). & Ocho & $\begin{array}{l}\text { Evitación, exploración, socialización, } \\
\text { actividad, compras de productos de moda, } \\
\text { exclusividad, servicio y estética }\end{array}$ \\
\hline Križan et al., (2018). & Dos & $\begin{array}{l}\text { Ubicación del centro comercial y atractivo del } \\
\text { centro comercial }\end{array}$ \\
\hline Upadhyaya (2017). & Siete & $\begin{array}{l}\text { Ambiente físico, Administración de } \\
\text { propiedades, Promociones, Experiencia de } \\
\text { compra, Entretenimiento, Disponibilidad y } \\
\text { Satisfacción y lealtad del cliente }\end{array}$ \\
& & $\begin{array}{l}\text { Ambiente físico, limpieza y seguridad; diseño } \\
\text { y ambiente natural; movilidad y accesibilidad } \\
\text { servicios añadidos y entretenimiento; y } \\
\text { variedad, calidad y prestigio de la oferta }\end{array}$ \\
\hline $\begin{array}{l}\text { Ortegón \& Royo } \\
\text { (2017). }\end{array}$ & Cinco & $\begin{array}{l}\text { Accesibilidad, condiciones de } \\
\text { estacionamiento, variedad comercial, } \\
\text { atmósfera del centro comercial }\end{array}$ \\
\hline $\begin{array}{l}\text { Teller, Wood \& Floh } \\
\text { (2016). }\end{array}$ & Cuatro & \\
\hline
\end{tabular}

\footnotetext{
${ }^{1}$ En scopus la ecuación de búsqueda corresponde a $<<$ ( TITLE-ABS-KEY ( attract*) AND TITLE-ABSKEY ( mall ) OR TITLE-ABS-KEY ( shopping AND center) ) AND ( LIMIT-TO ( DOCTYPE, "ar" ) ) AND ( LIMIT-TO ( SUBJAREA, "SOCI") OR LIMIT-TO ( SUBJAREA, "BUSI" ) >>

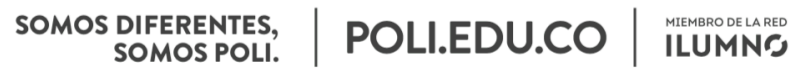




\begin{tabular}{|c|c|c|}
\hline Anselmsson (2016). & Siete & $\begin{array}{l}\text { Acceso, oferta, ambiente físico, servicio, } \\
\text { servicios alimenticios, promoción, } \\
\text { entretenimiento }\end{array}$ \\
\hline Kunc et al., (2016). & Seis & $\begin{array}{l}\text { Ubicación/Accesibilidad, estacionamientos, } \\
\text { clientes potenciales, tamaño del área rentable, } \\
\text { número y tipo de negocios, entretenimiento y } \\
\text { ocio. }\end{array}$ \\
\hline Makgopa (2016). & Cinco & $\begin{array}{l}\text { Comodidad de estacionamientos, acceso, } \\
\text { horario, conveniencia e higiene de los baños. }\end{array}$ \\
\hline $\begin{array}{l}\text { Kabadayi \& Paksoy } \\
\text { (2016). }\end{array}$ & Cinco & $\begin{array}{l}\text { Experiencia en visitas, orientado a objetivos } \\
\text { (variedad), socialización, ahorro de tiempo y } \\
\text { búsqueda de ofertas (ofertas) }\end{array}$ \\
\hline Wei \& Sim (2014). & Tres & $\begin{array}{l}\text { Confianza de la marca, afecto de marca y } \\
\text { percepción del centro comercial }\end{array}$ \\
\hline $\begin{array}{l}\text { Gilboa \& Vilnai-Yavetz } \\
\text { (2012). }\end{array}$ & Dos & Conveniencia y entretenimiento \\
\hline $\begin{array}{l}\text { González-Hernández \& } \\
\text { Orozco-Gómez (2012). }\end{array}$ & Seis & $\begin{array}{l}\text { Esencia del centro comercial, popularidad y } \\
\text { programas promocionales, servicio personal, } \\
\text { ambiente interno, opciones recreativas y } \\
\text { ambiente físico externo. }\end{array}$ \\
\hline Singh \& Sahay (2012). & Cinco & $\begin{array}{l}\text { Ambiente físico, la infraestructura física, el } \\
\text { enfoque de marketing, conveniencia y } \\
\text { seguridad }\end{array}$ \\
\hline Rajagopal (2011). & Seis & $\begin{array}{l}\text { Logística y servicios, atracciones del mercado, } \\
\text { preferencias de compra, relación con el } \\
\text { cliente, percepción de los compradores; y } \\
\text { excitación y alegría }\end{array}$ \\
\hline $\begin{array}{l}\text { Arslan, Sezer \& } \\
\text { Isigicok (2010). }\end{array}$ & Cinco & $\begin{array}{l}\text { Oferta, condiciones de confort, socialización } \\
\text { en un entorno seguro, accesibilidad, y ocio. }\end{array}$ \\
\hline Teller \& Elms (2010). & Diez & $\begin{array}{l}\text { Valor de oferta de productos, tipo de producto, } \\
\text { personal de venta, accesibilidad, } \\
\text { estacionamiento, mezcla de almacenes, mezcla } \\
\text { de pequeños negocios no almacenes, } \\
\text { atmósfera, orientación e infraestructura. }\end{array}$ \\
\hline Özsoy (2010). & Cinco & $\begin{array}{l}\text { Atractivo, entretenimiento y ocio, entorno } \\
\text { minorista, importancia de la ubicación, } \\
\text { socialización }\end{array}$ \\
\hline Rajagopal (2009) & Cinco & $\begin{array}{l}\text { Logística relacionada (conveniencia), } \\
\text { ambiente fisico, excitación y alegría } \\
\text { (entretenimiento), variedad de la oferta, y } \\
\text { promoción de ventas }\end{array}$ \\
\hline El-Adly, (2007). & Seis & $\begin{array}{l}\text { Confort, entretenimiento, diversidad, esencia } \\
\text { centro comercial, conveniencia y lujo }\end{array}$ \\
\hline
\end{tabular}




\begin{tabular}{lll}
\hline De Juan (2004). & Nueve & $\begin{array}{l}\text { Atuendo de consumidor, variedad de producto, } \\
\text { profesionalismo de vendedores, tranquilidad } \\
\text { durante el proceso de compra, limpieza, } \\
\text { facilidad de transición entre almacenes, } \\
\text { precios, estacionamiento y horario de atención }\end{array}$ \\
\hline $\begin{array}{l}\text { Martin \& Turley } \\
\text { (2004). }\end{array}$ & Ocho & $\begin{array}{l}\text { Factores ambientales físicos, factores de } \\
\text { diseño, variedad, deseo de permanecer, } \\
\text { emoción, involucramiento y motivación del } \\
\text { consumo (social y objetivo) }\end{array}$ \\
\hline $\begin{array}{l}\text { Gorter, Nijkamp \& } \\
\text { Klamer (2003). }\end{array}$ & Cinco & $\begin{array}{l}\text { Accesibilidad, estacionamiento, ambiente } \\
\text { físico, número de personas, tamaño de los } \\
\text { productos }\end{array}$ \\
\hline $\begin{array}{l}\text { Geisel, Narasimhan \& } \\
\text { Sen (1993). }\end{array}$ & Tres & $\begin{array}{l}\text { Tamaño del centro comercial, tiempo de viaje } \\
\text { y el atractivo del centro comercial }\end{array}$ \\
\hline
\end{tabular}

Fuente: Elaboración propia.

La revisión de literatura revelo que no existe un consenso respecto a la cantidad y tipología de factores que componen la atracción, sin embargo, se pueden deducir variables de atracción comunes, que, por su carácter repetitivo y semejante, pueden agruparse en una variable de interés frecuente como por ejemplo: a) la facilidad de acceso que incluye la ubicación, la señalización, la conveniencia de rutas, tiempo de viaje, conveniencia de horarios y facilidades de uso de estacionamientos; b) la oferta y variedad del centro comercial, que incluye la diversidad de tiendas, productos y servicios, preferencias de compra, promociones, valor de la oferta y personal de venta disponibles para los visitantes en el centro comercial incluyendo la sensación de lujo y prestigio debido a la presencia de tiendas, productos y servicios exclusivos; c) Presencia de clientes en el centro comercial, que incluye número de personas, características del consumidor, socialización, circulación y aglomeración de personas; d) Diseño físico del centro comercial, que contiene aspectos de percepción en términos de su tamaño, diseño exterior o interior, arquitectura, atractivo físico, condiciones de confort, limpieza y apariencia global agradable de la atmosfera, incluyendo ambiente de naturaleza en el centro comercial; y e) Entretenimiento del centro comercial, que contiene aspectos asociados a la respuesta de excitación, motivación, involucramiento y alegría del consumidor, debido a las opciones recreativas y de ocio disponibles. 
Finalmente se observa que hoy en día existe una gran variedad de factores de atracción de los entornos de comercio modernos, que se han propuesto y estudiado en diversas publicaciones de acuerdo con las necesidades y contextos específicos de cada investigación (para una revisión, véase Gomes \& Paula, 2017). En consecuencia, las futuras investigaciones tendrán que comprobar estos factores, y proponer nuevos factores alternativos que sean capaces de demostrarse siguiendo el método científico en línea con las tendencias de producción de la literatura de marketing centrada en mejorar la medición de la experiencia y memorabilidad de visita (Flacandji \& Krey, 2020).

\section{REFERENCIAS}

Aliagha, G. U., Qin, Y. G., Ali, K. N., \& Abdullah, M. N. (2015). Analysis of Shopping Mall Attractiveness and Customer Loyalty. Journal Teknologi, 74(2), 15-21.

Anselmsson, J. (2016). Effects of shopping centre re-investments and improvements on sales and visit growth. Journal of Retailing and Consumer Services, 32, 139-150.

Arslan, T. V., Sezer, F. S., \& Isigicok, E. (2010). Magnetism of shopping malls on young Turkish consumers. Young Consumers: Insight and Ideas for Responsible Marketers, 11(3), 178-188.

Bäckström, K. (2011). Shopping as leisure: An exploration of manifoldness and dynamics in consumers shopping experiences. Journal of Retailing and Consumer Services, 18(3), 200-209.

Calvo-Porral, C., \& Lévy-Mangín, J. P. (2018). Pull factors of the shopping malls: an empirical study. International Journal of Retail \& Distribution Management, 46(2), 110-124.

De Juan, M. D. (2004). Why do people choose the shopping malls? The attraction theory revisited: A Spanish case. Journal of International Consumer Marketing, 17(1), 7196.

El-Adly, M. I. (2007). Shopping malls attractiveness: a segmentation approach. International Journal of Retail \& Distribution Management, 35(11), 936-950.

El-Adly, M. I., \& Eid, R. (2015). Measuring the perceived value of malls in a non-Western context: the case of the UAE. International Journal of Retail \& Distribution Management, 43(9), 849-869.

Elmashhara, M. G., \& Soares, A. M. (2019). The impact of entertainment and social interaction with salespeople on mall shopper satisfaction. International Journal of Retail \& Distribution Management. Vol. 47 No. 2, pp. 94-110.

Flacandji, M., \& Krey, N. (2020). Remembering shopping experiences: the shopping experience memory scale. Journal of Business Research, 107, 279-289.

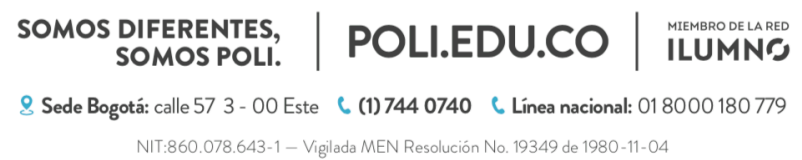


Frasquet, M., Vallet, T., \& Gil, I. (2002). Key factors in shopping centre management: evidence from Spain. The International Review of Retail, Distribution and Consumer Research, 12(4), 337-354.

Geisel, M. S., Narasimhan, C., \& Sen, S. K. (1993). Quantifying the competitive impact of a new entrant. Journal of Business Research, 26(3), 263-277.

Gilboa, S., \& Vilnai-Yavetz, I. (2012). Segmenting multicultural mall visitors: the Israeli case. Marketing Intelligence \& Planning, 30(6), 608-624.

Gomes, R. M., \& Paula, F. (2017). Shopping mall image: systematic review of 40 years of research. The International Review of Retail, Distribution and Consumer Research, 27(1), 1-27.

González-Hernández, E. M., \& Orozco-Gómez, M. (2012). A segmentation study of Mexican consumers based on shopping centre attractiveness. International Journal of Retail \& Distribution Management, 40(10), 759-777.

Gorter, C., Nijkamp, P., \& Klamer, P. (2003). The attraction force of out-of-town shopping malls: a case study on run-fun shopping in the Netherlands. Tijdschrift voor economische en sociale geografie, 94(2), 219-229.

Han, H., Sahito, N., Thi Nguyen, T. V., Hwang, J., \& Asif, M. (2019). Exploring the features of sustainable urban form and the factors that provoke shoppers towards shopping malls. Sustainability, 11(17), 4798.

Hu, H., \& Jasper, C. R. (2018). Understanding the Shopping Experience and Its Implications for Malls as Marketing Media: Attracting and Retaining Customers through Fashion, Service, and Improved Food Options. Journal of Advertising Research, 58(2), 151-164.

Kabadayi, S., \& Paksoy, B. (2016). A segmentation of Turkish consumers based on their motives to visit shopping centres. The International Review of Retail, Distribution and Consumer Research, 26(4), 456-476.

Križan, F., Bilková, K., Barlík, P., Kita, P., \& Kita, P. (2018). Spatial Distribution of Consumer Preferences: Case Study of Shopping Malls in Bratislava. Theoretical and Empirical Researches in Urban Management, 13(1), 13-21.

Lucia-Palacios, L., Pérez-López, R., \& Polo-Redondo, Y. (2020). Does stress matter in mall experience and customer satisfaction?. Journal of Services Marketing. Vol. 34 No. 2, pp. 177-191

Makgopa, S. (2016). Determining shopping mall visitors' perceptions on mall attributes. Problems and Perspectives in Management, 14(3-2), 3-2.

Martin, C. A., \& Turley, L. W. (2004). Malls and consumption motivation: an exploratory examination of older Generation Y consumers. International Journal of Retail \& Distribution Management, 32(10), 464-475.

Merrilees, B., Miller, D., \& Shao, W. (2016). Mall brand meaning: an experiential branding perspective. Journal of Product \& Brand Management, 25(3), 262-273. 
Ortegón Cortázar, L., \& Royo Vela, M. (2017). Attraction factors of shopping centers: Effects of design and eco-natural environment on intention to visit. European Journal of Management and Business Economics, 26(2), 199-219.

Ortegón, L. \& Royo, M., (2015). Brand image and lexicographic analysis: an application to shopping malls. Cuadernos de Gestión, 15 (2), 143-162

Ortegon-Cortazar, L. (2019). Role of the eco-natural environment as an alternative attractiveness factor in malls. Management Research: Journal of the Iberoamerican Academy of Management. Vol. 17 No. 2, pp. 168-186.

Ortegón-Cortázar, L., \& Royo-Vela, M. (2019). Effects of the biophilic atmosphere on intention to visit: the affective states' mediating role. Journal of Services Marketing. Vol. 33 No. 2, pp. 168-180

Özsoy, M. (2010). User preferences on transformations of shopping centers into private urban public spaces: The case of Izmir, Turkey. African Journal of Business Management, 4(10), 1990.

Palacios, L., Pérez, R., \& Polo, R., (2016). Cognitive, affective and behavioural responses in mall experience: A qualitative approach. International Journal of Retail \& Distribution Management, 44(1), 4-21.

Rajagopal. (2009). Growing shopping malls and behaviour of urban shoppers. Journal of Retail \& Leisure Property, 8(2), 99-118.

Rajagopal. (2011). Determinants of shopping behavior of urban consumers. Journal of International Consumer Marketing, 23(2), 83-104.

Roux, T., Mahlangu, S., \& Manetje, T. (2020). Digital signage as an opportunity to enhance the mall environment: a moderated mediation model. International Journal of Retail \& Distribution Management. Vol. ahead-of-print No. ahead-of-print.

Singh, H., \& Sahay, V. (2012). Determinants of shopping experience: exploring the mall shoppers of national capital region (NCR) of India. International Journal of Retail \& Distribution Management, 40(3), 235-248.

Teller, C., \& Elms, J. (2010). Managing the attractiveness of evolved and created retail agglomerations formats. Marketing Intelligence \& Planning, 28(1), 25-45.

Teller, C., Wood, S., \& Floh, A. (2016). Adaptive resilience and the competition between retail and service agglomeration formats: an international perspective. Journal of Marketing Management, 32(17-18), 1537-1561.

Upadhyaya, M. (2017). Variables affecting customer behaviour and its effect on commitment towards shopping hubs of Gurgoan city. Journal of Business and Retail Management Research, 11(3).

Wei Khong, K., \& Sim Ong, F. (2014). Shopper perception and loyalty: a stochastic approach to modelling shopping mall behaviour. International Journal of Retail \& Distribution Management, 42(7), 626-642. 
